\title{
TÉCNICA ASÉPTICA DEL PASO DE SONDA VESICAL
}

Paola Andrea Diaz Lozano, Nidia Gabriela Garzon Mojica, Carlos Fredy Infante, Sergio Frederik Piratoba, Johana Catalina Rodriguez Clavijo, Lady Heliana Rodriguez Lopez**

\section{Resumen}

Se investigaron los conocimientos, actitudes y prácticas del personal de enfermería en el manejo de la técnica aséptica durante el paso de sonda vesical en un hospital de tercer nivel de complejidad, con base en el protocolo que tiene la institución sobre el procedimiento. El trabajo se enmarcó dentro de los parámetros de la investigación cualitativa, tomando como referencia el modelo de conocimientos, actitudes y prácticas (CAP). Para ello se elaboró una encuesta que una vez validada se aplicó a 24 enfermeras y 67 auxiliares que laboran en los servicios de atención al paciente adulto y los datos fueron procesados utilizando el programa SPSS. Para cada uno de los ítems se hizo el análisis comparativo enfermero-auxiliar de enfermería, teniendo en cuenta la diferencia en el nivel de capacitación. Los resultados generales reportaron que el $90.1 \%$ del total de encuestados conoce la existencia del protocolo y un $18.7 \%$ no lo ha leído en forma detenida. La comparación mostró un mejor nivel de conocimientos y actitudes en los profesionales, pero mejores prácticas en los auxiliares. La población encuestada en su totalidad considera que utilizar la técnica aséptica durante el paso de sonda vesical es indispensable para evitar posibles infecciones, lo cual permite suponer una actitud positiva frente al procedimiento. En general hay un buen nivel de conocimientos sobre la técnica, pero hay déficit en cuanto a qué hacer en situaciones especiales a pesar de que se encuentran contempladas en el capítulo de recomendaciones del protocolo del hospital.

\section{Introducción}

El paso de sonda vesical es un procedimiento en apariencia sencillo, que se realiza con fines terapéuticos o diagnósticos y requiere la aplicación estricta de una técnica aséptica. En la institución objeto de estudio se encuentra establecido un protocolo para dicho procedimiento; el grupo de investigación, con el objeto de determinar los conocimientos, actitudes y prácticas del personal de enfermería profesional y auxiliar en el manejo de la técnica aséptica durante el paso de sonda vesical, realizó un trabajo de investigación cualitativa utilizando el modelo CAP. Para ello diseñó, validó y aplicó una encuesta, basada en el protocolo, a 24 profesionales y 67 auxiliares, cuyos resultados fueron procesados en el programa SPSS. El análisis se hizo comparando las profesiones de enfermería y auxiliares, estableciendo las categorías: alta, si las respuestas positivas superaban el $80 \%$, media, si se encontraban entre $60-80 \%$ y baja si era menor del $60 \%$. Con estos

\footnotetext{
Estudiantes de VIII Semestre de Enfermería, Fundación Universitaria de Ciencias de la Salud, Hospital de San José.

Este trabajo fue realizado bajo la tutoría de la Lic. Ana Julia Carrillo A. Directora de Posgrados de Enfermería, Profesora Asociada, Fundación Universitaria de Ciencias de la Salud.
}

parámetros, se encontró que los conocimientos se encuentran en un nivel alto, la actitud es positiva y la práctica está en el nivel medio. Este último dato es relevante teniendo en cuenta que genera factores de riesgo de infección al paciente.

\section{Materiales y métodos}

Para dar respuesta a la pregunta de investigación, el trabajo se enmarcó en el método cualitativo, utilizando el modelo de investigación de conocimientos, actitudes y prácticas (C.A.P), el cual busca comparar dos situaciones, una ideal, para este caso la aplicación del protocolo y una real, con el fin de verificar el comportamiento del grupo objeto de estudio.

Se tomó como muestra la totalidad del personal de enfermería que labora en los servicios de adultos de la institución, en total 103 personas. Se excluyeron 12 por no cumplir los criterios de inclusión (ser personal de planta y pertenecer al servicio por un período mayor de un mes, sin interesar el turno). Se encuestaron 91 personas, 24 profesionales y 67 auxiliares en los servicios de atención al paciente adulto de un hospital de tercer 
nivel, durante el período comprendido entre el 1 y 30 de marzo de 2004.

La encuesta se elaboró basada en el protocolo, clasificando las preguntas en tres categorías, conocimientos, actitudes y prácticas, clasificación conocida solo por el grupo investigador.

\section{Resultados}

Se observó que el 90,1\% conocen la existencia del protocolo, el $16,7 \%$ de los enfermeros y el $19,4 \%$ de los auxiliares no lo han leído en forma detenida, lo que indica que no todo el personal tiene clara la forma adecuada del procedimiento según el protocolo (Figura 1). Se aprecia que la mayoría conoce la definición de técnica aséptica: 95,8\% enfermeros y 92,5\% auxiliares. Se confunden con facilidad ante el concepto de antisepsia ya que no tienen clara la definición de séptico (25,0\% de los enfermeros y $29,9 \%$ de los auxiliares contestaron incorrectamente). Se resalta que la mayoría de los encuestados saben que el paso de sonda vesical debe ser un procedimiento estéril, pero se observa que el $4,2 \%$ de los profesionales y el $6 \%$ de los auxiliares refieren que debe ser un procedimiento limpio, lo cual indica que un porcentaje bajo no tiene clara la importancia de la técnica aséptica. De los encuestados, conocen la ubicación del meato urinario con precisión el 91,7\% de los enfermeros y el 89,6\% de los auxiliares. Sin embargo, llama la atención la práctica que realizan cuando

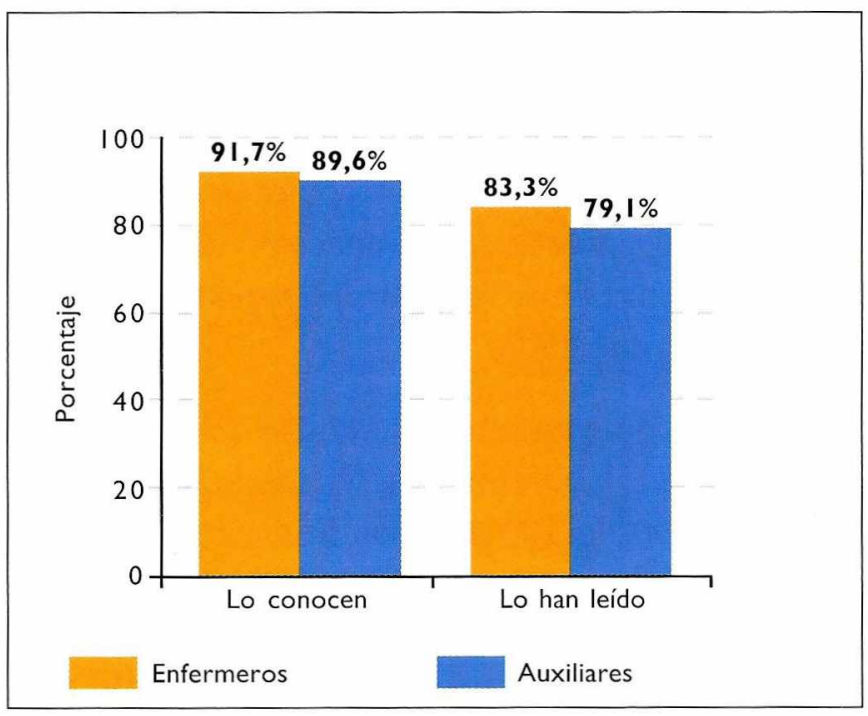

Figura I. Porcentajes comparativos entre enfermeros y auxiliares en cuanto al conocimiento y lectura del protocolo. se presenta confusión en la identificación del meato urinario con el orificio vaginal, cambiando la sonda en un $95,8 \%$ los enfermeros y un $62,7 \%$ los auxiliares. Además, un $32,8 \%$ de los auxiliares no realiza la práctica correcta incumpliendo el protocolo y poniendo en riesgo la integridad de la paciente (Figura 2).

El 58,3\% de los enfermeros y el 59,7\% de los auxiliares no taponan la vagina antes de realizar el procedimiento; es preocupante que la mayoría del personal prefiere realizarlo sin tener contacto con fluidos, en un 79,2\% los enfermeros y un 73,1\% los auxiliares, desconociendo el paso que señala el protocolo al realizar un sondaje vesical a una mujer que presenta sangrado o flujo vaginal.

En cuanto a la actitud, el 62,5\% de los enfermeros y el 86,6\% de los auxiliares considera que la labor que desempeña llena por completo su satisfacción personal. Sin embargo, llama la atención que un considerable número de enfermeras manifiestan inconformidad con su labor por diferentes circunstancias. El sentimiento más frecuente al realizar un sondaje vesical es de satisfacción laboral, lo cual se convierte en un factor positivo para la relación enfermero paciente. Los sentimientos negativos, como vergüenza y ansiedad, se presentaron con una incidencia baja demostrando así la buena calidad del cuidado con miras al adecuado ejercicio de la profesión. Otros sentimientos manifestados como pudor, pesar, indiferencia y lástima son el resultado determinado por la experiencia individual.

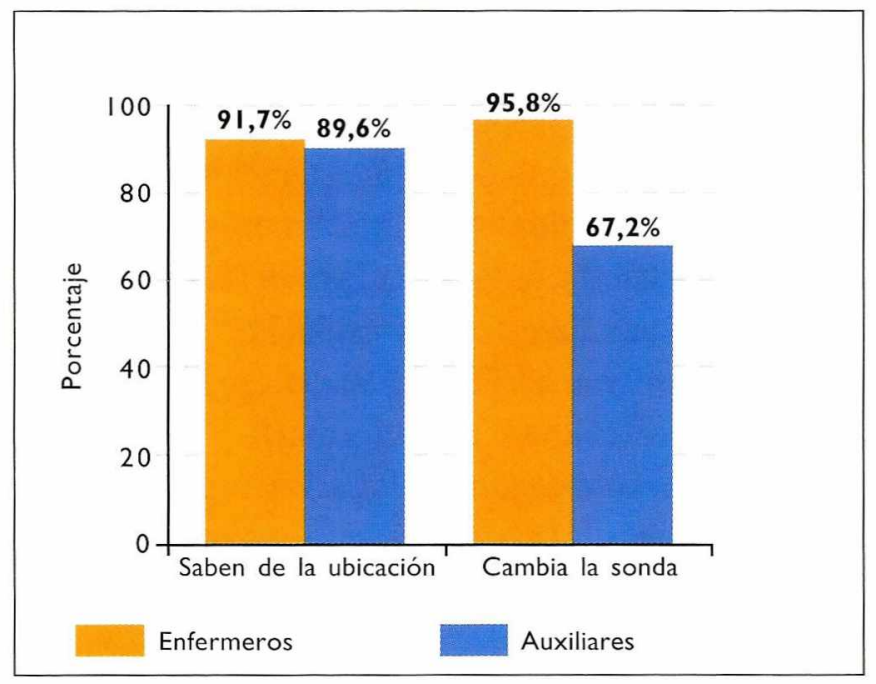

Figura 2. Porcentajes comparativos entre enfermeros y auxiliares sobre la ubicación del meato urinario y práctica en caso de confundirlo con el orificio vaginal. 
El 100\% de los enfermeros y el $97 \%$ de los auxiliares considera que al seguir una técnica aséptica durante el paso de sonda vesical, debe cumplirse para evitar posibles infecciones, lo cual genera una actitud positiva frente al procedimiento (Figura 3).

Un punto importante en el cual la población arrojó resultados inesperados es en la necesidad de obtener el consentimiento informado. Aunque la mayoría lo hace antes del procedimiento, que es lo indicado, un $15,4 \%$ lo realiza antes y después, lo cual demuestra que no hay seguridad en el momento en que debe obtenerse y genera una pérdida de tiempo al repetir innecesariamente un procedimiento. Además, si el paciente llegara a negar el consentimiento informado para la realización de un procedimiento, el 72,2\% de los enfermeros y el 80,1\% de los auxiliares prefieren informar al superior. El 83,3\% de los enfermeros y el 67,2\% de los auxiliares tratan de convencer al paciente, observando que es la actitud apropiada de acuerdo con lo establecido.

La encuesta mostró que el personal al encontrar abierto el paquete de una sonda, en primer lugar informa a la farmacia, lo que indica una buena actitud frente al cuidado del paciente y al control de los insumos recibidos. Otro sentimiento positivo que demostró el personal es el de desechar la sonda y no realizar el procedimiento ya que el $100 \%$ de los enfermeros cumplen en forma estricta con los parámetros establecidos. Sin embargo, un $10.4 \%$ de los auxiliares omite esta práctica utilizan-

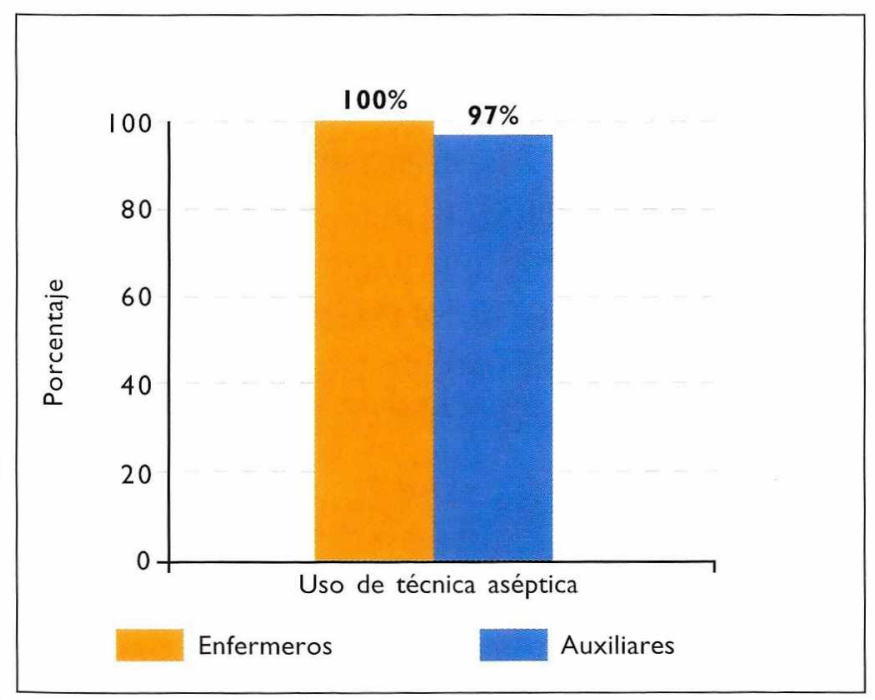

Figura 3. Porcentaje comparativo entre enfrmeros y auxiliares en cuanto al uso de técnica aséptica para evitar posibles infecciones. do la sonda para otro procedimiento sin tener en cuenta el protocolo, aumentando riesgos al paciente (Figura 4). El $25 \%$ de los enfermeros y el $52,2 \%$ de los auxiliares que no desechan la sonda si encontraran el paquete abierto, indica que no tienen clara la importancia del control de los elementos estériles, que cuando fallan deben eliminarse del servicio para evitar que sean utilizados en otros pacientes.

Al observar una persona que no introduce la sonda como lo exige el protocolo, los enfermeros en un $87,5 \%$ y los auxiliares en un $88,1 \%$ la corrigen sin que el paciente se de cuenta, actitud positiva ya que se evita exponer al enfermo a un mal procedimiento y no lesiona la imagen del colaborador. Un 45,8\% de los enfermeros y un $25,4 \%$ de los auxiliares lo corrige durante y después del procedimiento. Al hacerlo durante, permite que la persona no recurra en la misma falta y corregirlo después permite analizar la situación y reforzar sus conocimientos para mejorar la actitud frente al paciente.

El personal en general identificó los datos para registraren la hoja de enfermería, pero nos llamó la atención que un 13,18\% no considera necesario anotarlos, actitud negativa que los puede perjudicar, pues la hoja de enfermería es el documento legal en el cual obligatoriamente se debe describir lo que se realiza, protegiéndolos de cualquier implicación legal o laboral. La actitud que demostró el personal es adecuada para mejorar la calidad y el buen cuidado del paciente.

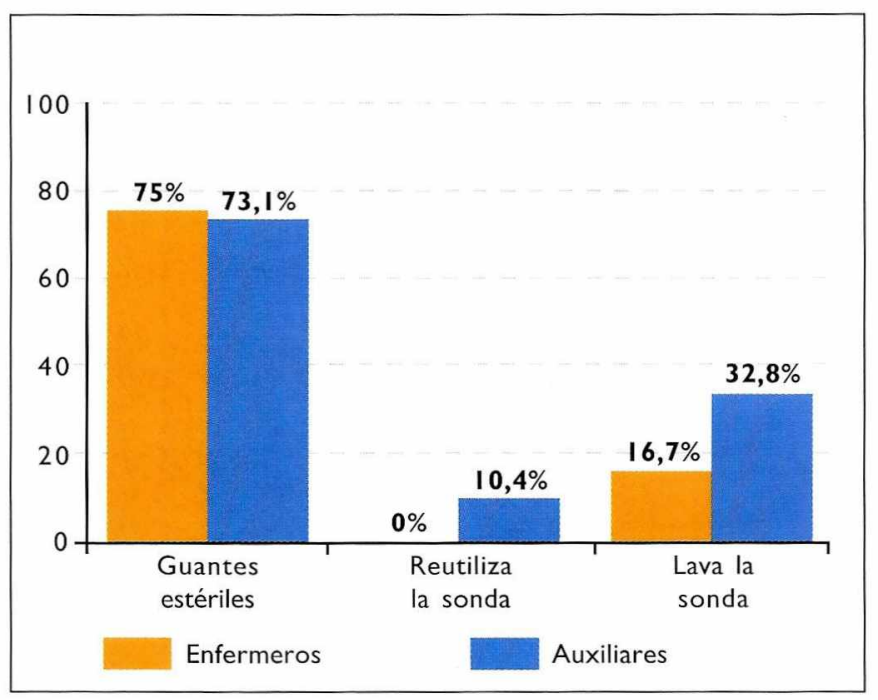

Figura 4. Porcentajes comparativos entre enfermeros y auxiliares para determinar la utlización de la técnica aséptica al paso de sonda vesical. 
En relación con la práctica, se encontró en un nivel medio debido a la poca frecuencia del paso de sonda vesical en algunos pabellones del Hospital de San José y también por la falta de aplicación de los conocimientos. Se observó, por ejemplo, que el $100 \%$ de los enfermeros y un $92,5 \%$ de los auxiliares utiliza guantes estériles, pero es preocupante que el $10,4 \%$ de los auxiliares utiliza guantes limpios para este procedimiento. El baño genital externo en la mujer lo realiza en forma correcta el 95,8\% de enfermeros y el 86,6\% de auxiliares. Aunque la mayoría contestaron que lo hacen de arriba hacia abajo, un porcentaje llamativo de $16,7 \%$ de enfermeros y un $10,4 \%$ de auxiliares se confunden realizándolo del centro a la periferia.

Los enfermeros en un 75\% y auxiliares en un 73,1\% después de realizado el baño genital utilizan guantes estériles, pero es preocupante que un $22 \%$ de la población después de realizar el baño genital se coloque guantes limpios sin seguir los pasos que exige el protocolo, demostrando incoherencia entre la teoría y la práctica. Cuando encuentran obstrucción al pasar la sonda vesical, lo usual en el $75 \%$ de enfermeros y $64,2 \%$ de los auxiliares es retirar la sonda, siendo la acción adecuada. Otras opciones como: retirar y aplicar más lubricante y retirar un poco e introducirla, alcanzó un porcentaje importante en los enfermeros $(41,7 \%)$ y auxiliares $(35,8 \%)$, pero lo más grave de este resultado es que un $9 \%$ de los auxiliares continúa el procedimiento sin importar el daño que podría causarle al paciente (Figura 5).

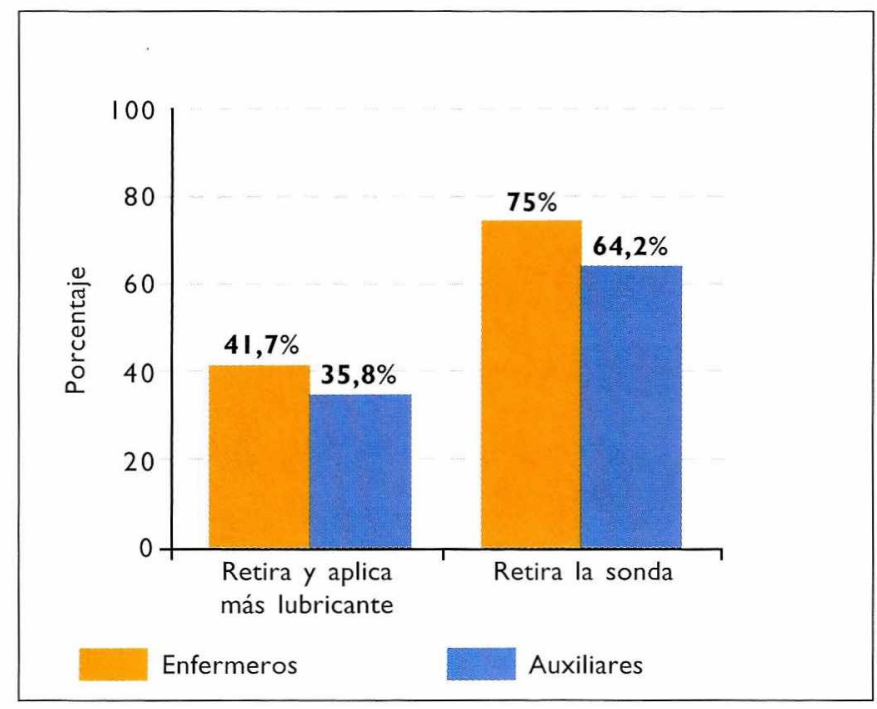

Figura 5. Porcentajes comparativos entre enfermeros y auxiliares al encontrar obstrucción que dificulta el paso de sonda vesical.
Para todos los enfermeros $(100 \%)$ es clara la ubicación del cystofló por debajo de la vejiga al lado del paciente, pero preocupa que el 10,4\% de los auxiliares lo coloca a la misma altura al lado del enfermo, el 19,4\% de los mismos lo pone en el suelo y solo el $85,1 \%$ lo hace de manera correcta. Se observa que los conocimientos adquiridos durante sus estudios y experiencia no se relacionan con la práctica. Dado que la literatura describe el alto riesgo de infección urinaria cuando hay retorno de orina contaminada, es un punto importante que se debe tener en cuenta en el momento de implementar medidas de mejoramiento continuo.

\section{Conclusiones}

A pesar de que el $90 \%$ del personal conoce el protocolo del hospital para el paso de sonda vesical, sólo el $80 \%$ lo ha leído completo. En general, hay un buen nivel de conocimientos sobre la técnica aséptica para el paso de sonda vesical, pero hay déficit en saber qué hacer en situaciones especiales que se encuentran contempladas en el capítulo de recomendaciones del protocolo del hospital.

La satisfacción personal es mayor en el personal auxiliar que en los enfermeros, lo cual puede incidir en la aplicación de los conocimientos o la práctica. La población encuestada en general está convencida de la necesidad de la técnica aséptica en al paso de sonda vesical, lo cual genera una actitud positiva frente al procedimiento y se refleja en los resultados que muestran coherencia entre los conocimientos y la práctica.

La encuesta reportó un nivel alto en conocimientos y actitudes, pero no llegó al 100\%. En la práctica solo alcanzó el nivel medio, que llama la atención porque genera riesgos potenciales al paciente. Se recomienda hacer un monitoreo permanente del procedimiento hasta que se logre el $100 \%$ de práctica correcta.

\section{Lecturas recomendadas}

- Castellote García M de J. Protocolo de Enfermería, sondaje Vesical.(seriado en línea) 2004 Mar. (Disponible en: http:www.sourfear.opolanco.com/Apat/boletin9/sonda.htm. (acceso 3 de marzo de 2004).

- Enciclopedia de la Psicologia y la Pedagogia. Vol 7. Diccionario de Psicología. Madrid : Sedmary, 1980. 
- Fundación Santa Fé Bogotá, Asepsia y antisepsia en lavado de manos clínico. Actuali Enf. 2002; Vol. 5 (3): 1-3.

- Gutierrez Fanti J. Ciencia, conocimiento y método científico. 2004 Mar 3 (monografía en Internet). Disponible en: http: // www.monografias.com/trabajos/epistemología $2 / \mathrm{shtml}$.

- Hospital de San José, Bogotá. Comité de Infecciones. Manuel de Prevención y control de infecciones intrahospitalarias. Bogotá: Hospital de San José, s.f.

- Maden Styles M. El Consejo Internacional de Enfermería y la reglamentación : modelos para el siglo XXI. Bogotá : CIE, 1996. p. 350.

- Márquez Pérez M. Satisfacción laboral. 2004 abr. 8. Disponible en:http:// www.gestiopolis.com/recursos/expert/ capsexp/pagans/rh/36/satlabo.htm.

- Mateo T P. Protocolo de Enfermería Cuidados en el Sondaje Vesical. (se encuentra en la dirección (seriado en línea) 2004 mar 2 disponible en http: // www.opolanco.es/Apat/ Boletin12/sondas.htm\#cuidados.

- Rivera C. La vergüenza como virtud Civil (seriado en línea) 2004 abr.9 disponible en http//www.elpelado.com/letras.index.
- Rodríguez Vega A. et.al. Infección urinaria nosocomial y la prevención de la infección urinaria asociada a catéter vesical. Seriado en línea 2004 abr 9 disponible en (tp: // www. Encolombia.com/medicina/402infección.

- Rojas Vidal, M. del Coicou A. Asesoría y Proyectos de Investigación. (monografía en Internet). Disponible en: http: // www.sepsiquiatria.org/sepsiquiatria/html/información_sociedad/ manual/a 5n5htm.

- Torres G. J. Conocimientos, actitudes y prácticas del lavado de manos clínico por el personal de enfermería del servicio de urgencias de una institución hospitalaria de tercer nivel. Bogotá, 2002, 170 p. Trabajo de Grado (Enfermero). Pontificia Universidad Javeriana. Facultad de Enfermería.

- Universidad Nacional de Colombia. Sede Bogotá. Facultad de Enfermería. Grupo de Cuidado. El arte y la ciencia del cuidado. Bogotá: UNAL, 2002, 387 p.

- Varela Cota O Hervé. Definición de ansiedad. Seriado (articulo de Internet). 2004 mar 2 disponible en http: // www. Sicopedagogia.com/definición/ansiedad

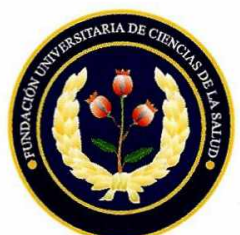

FUNDACIÓN UNIVERSITARIA DE CIENCIAS DE LA SALUD HOSPITAL DE SAN JOSÉ PROGRAMA INNOVADOR EN EL ÁREA DE LA SALUD

INFORMES:

Hospital de San José, entrada oriental, carrera 18 No. 8 - 95

Edificio Docente - Bogotá D.C. PBX: 5998977

Línea Nacional Gratuita: 018000113827

www. fucsalud.edu.co

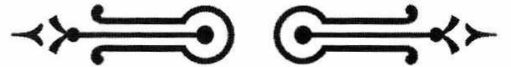

\section{WITOHISTOLOLGÍA}

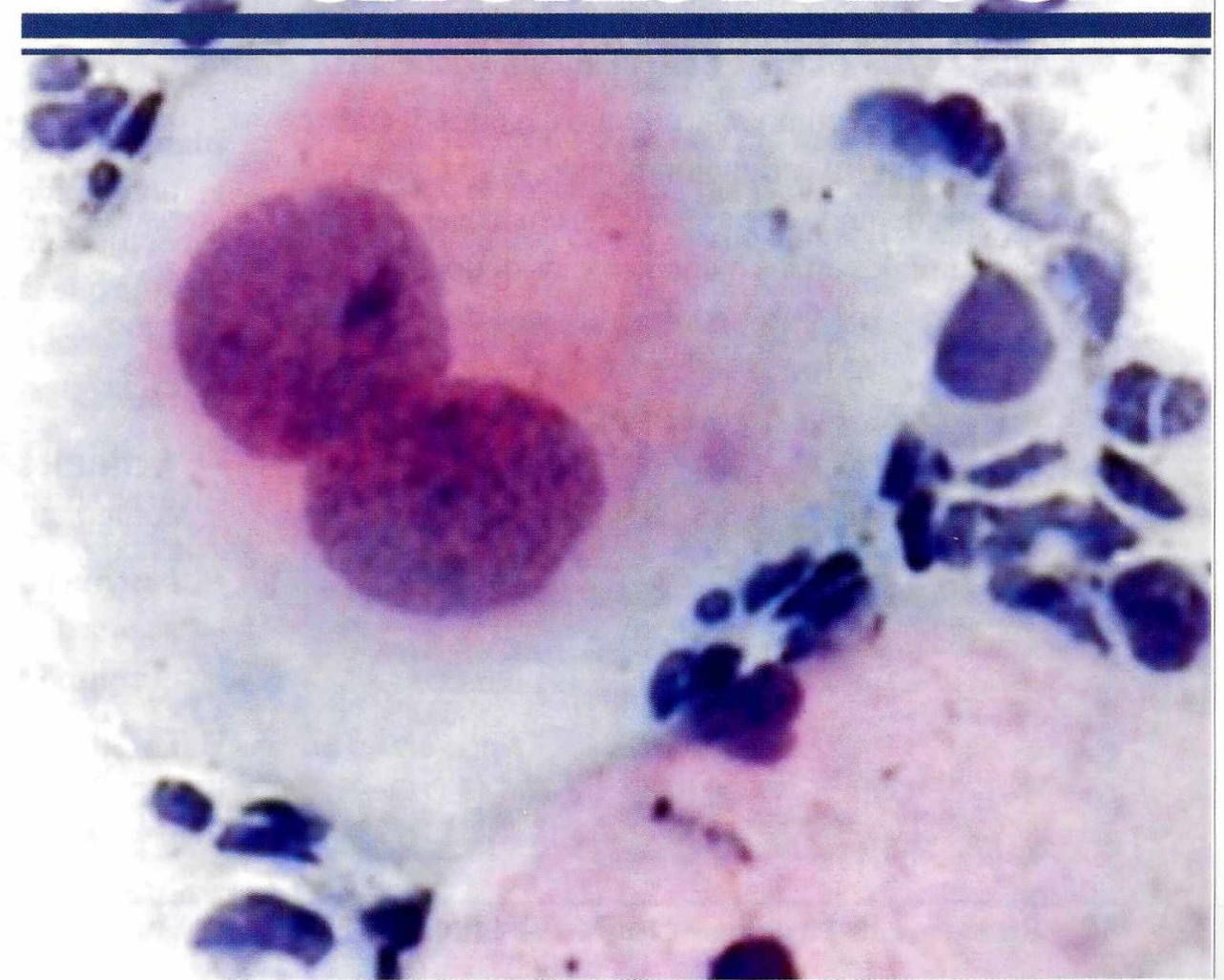

